\title{
Effect of New Generation Ready-Mix Herbicide against Complex Weed Flora in Transplanted Paddy and its Residual Effect on Succeeding Black Gram Crop
}

\author{
M. Shivashenkaramurthy*, Roopa S. Patil, M. J. Manju, \\ K. S. Krishna and Annapurna Neeralagi
}

ICAR-Krishi Vigyan Kendra, Sirsi-581401, Uttara Kannada, Karnataka, India

*Corresponding author

\begin{tabular}{l} 
K e y w o r d s \\
$\begin{array}{l}\text { Fenoxaprop,Pyrazos } \\
\text { ulfuron Ethyl, } \\
\text { Pretilachlor, Bio- } \\
\text { efficacy Monocot } \\
\text { and Paddy }\end{array}$ \\
\hline Article Info \\
$\begin{array}{l}\text { Accepted: } \\
14 \text { April } 2021 \\
\text { Available Online: } \\
\text { 10 May } 2021\end{array}$ \\
\hline
\end{tabular}

A B S T R A C T
An experiment was conducted to evaluate the Bio-efficacy and Phyto-toxicity of Fenoxaprop $6 \%$ + Pyrazosulfuron Ethyl 1.5\% + Pretilachlor 50\% ME (readymix)against monocot weeds and dicot weeds in paddy at Krishi Vigyan Kendra, Sirsi of University of Agricultural Sciences, Dharwad during Kharif 2018 and Kharif 2019. This new ready-mix herbicide was tried at different concentrations $(800,1000$ and $1200 \mathrm{ml} / \mathrm{ha}$ ) and compared with other herbicides viz.,Check I -Chlorimuron Ethyl 25\% WP @ 24g/ ha., Check II-Fenoxaprop-p-ethyl 9.3\% w/w EC @ 625 ml/ha., Check III- Pretilachlor 50\% EC @ 1500 ml/ha and Check IV- Pyrazosulfuron ethyl 10\% WP@150 g/ha. These herbicide treatments were compared with hand weeding, weed free and untreated control treatments. Fenoxaprop 6\% + Pyrazosulfuron Ethyl $1.5 \%$ + Pretilachlor 50\% ME (ready-mix)@ all the doses recorded lower weed dry weight as compare to rest of the treatments. Among chemical treatments, Fenoxaprop $6 \%$ + Pyrazosulfuron Ethyl 1.5\% + Pretilachlor 50\% ME (Readymix)@ 1200 ml/ha recorded the lowest weed dry weight followed by Fenoxaprop 6\% + Pyrazosulfuron Ethyl 1.5\% + Pretilachlor 50\% ME (ready-mix) @ 1000 ml/ha.Weed Free check recorded highest grain yield followed by Fenoxaprop 6\% + Pyrazosulfuron Ethyl 1.5\% + Pretilachlor 50\% ME (Readymix)at $1200 \mathrm{ml} / \mathrm{ha} \& 1000 \mathrm{ml} / \mathrm{ha}$ during both the seasons. Novisual symptoms of injury or phytotoxicity of Fenoxaprop $6 \%+$ Pyrazosulfuron Ethyl 1.5\% + Pretilachlor 50\% ME (ready-mix) were observed in even higher concentration of $3000 \mathrm{ml} / \mathrm{ha}$ on transplanted Paddy.

\section{Introduction}

Rice (Oryza sativa $\mathrm{L}$.) is the leading cereal of the world (Juraimi et al., 2013). Rice in India, contributing to about $40 \%$ of total food grain production. Weeds remove a large amount of nutrients from soil. An estimate shows that weeds can deprive the crops by $47 \% \mathrm{~N}, 42 \%$ $\mathrm{P}, 50 \% \mathrm{~K}, 39 \% \mathrm{Ca}$ and $24 \% \mathrm{Mg}$ of their nutrient uptake as well as reduce the yield potential by harboring number of crop pests (Balasubramaniyan and Palaniappan, 2001). 
Weed management always plays a vital role to achieving rice yield. Hence successful weed control is essential for obtaining optimum yield of rice. This can be achieved by removing weeds manually, mechanically and through chemical sprays or by their combinations. Manual weeding is although effective and most common method, however, scarcity and high wages of labour particularly during peak period of agricultural operations make this method uneconomic. Further, mechanical method of weed management is also time taking, cost intensive, much tedious and also does not remove all the weeds. Herbicidal weed management becomes a competitive and promising way to control weeds in transplanted rice, at least for first few weeks after transplanting of crop. The use of herbicides, therefore appears to be the only alternative (Alstorm, 1990) and in the present context, it is most preferable. Weed management in transplanted rice through herbicide application may be the best suited option.

Pre-emergence herbicides like butachlor, pretilachlor, anilofos and oxadiargyl are most commonly used for the control of weeds in transplanted rice. Bispyribac-sodium is also being recently used as early post-emergence control of weeds in transplanted rice (Yadav et al., 2009).About 350 species in more than 150 genera and 60plant families have been reported as weeds of rice(Barret and Seaman, 1980). Smith (1981) reported Poaceae as the most important weed family in rice. According to Joy et al., (1991) weed flora in rice consisted of $37 \%$ grasses $33 \%$ sedges and $30 \%$ broad leaved weeds. Chemical weed control is becoming popular and a number of post emergent herbicides differing in selectivity and mode of action. But single application of one herbicide is not that effective against complex weed flora throughout the crop season. Moreover, some of the grassy weeds, broad-leaf weeds and sedges are not effectively controlled by alone application of these herbicides. To achieve satisfactory control of complex weed flora, farmers resort to use 2,4-D, metsulfuron + chlorimuron or ethoxysulfuron as sequential post-emergence herbicides (Yadav et al.,2018). However, this adds to the cost of weed management. Being easy to apply, farmers' preference otherwise also remains mostly in favour of pre-emergence herbicides to achieve effective weed management at an early stage. Under such situations, more suitable option would be single shot application of ready-mix or tank-mix combination of herbicides as early post emergence.

Avudaithai and Veerabadran (2000) reported that combined application of different herbicides even at lower doses proved more effective against a broad spectrum of weeds. One of the recent ways to overcome the shift in weed flora and to prevent the development or delay the development of herbicide resistance in weeds is the use of herbicide mixtures and use of herbicides in rotation. Herbicide rotation means rotational use of herbicides with different mode of action. Herbicide mixtures will help to prevent the resistance problem and shift in weed population, which is always a problem associated with the use of single herbicide (Duary et al., 2015). Keeping this in view, an investigation was conducted to evaluate the bio-efficacy of Fenoxaprop $6 \%+$ Pyrazosulfuron Ethyl 1.5\% + Pretilachlor 50\% ME (readymix)as early post-emergence application against complex weed flora in transplanted rice and also its phytotoxicity on paddy and residual effects on succeeding crop of black gram.

\section{Materials and Methods}

An experiment was conducted to evaluate the Bio-efficacy of Fenoxaprop $6 \%+$ 
Pyrazosulfuron Ethyl 1.5\% + Pretilachlor 50\% ME (ready-mix)against Monocot weeds i.e. Echinochloa crusgalli, Echinochloa colonum, Ludwigia parviflora, Cyperus rotundus, Cyperus difformis, Cyperus iria, Fimbristylis miliacae, Monochoria vaginalis, Leptochloa chinensis \& Panicum repens and Dicot weeds i.e. Chenopodium album, Commelina benghalensis \& Eclipta alba in transplanted Paddy crop at Sirsi during Kharif 2018-19 \& Kharif, 2019-20.

Fenoxaprop 6\% + Pyrazosulfuron Ethyl 1.5\% + Pretilachlor 50\% ME (ready-mix)was evaluated for its bio-efficacy on weed flora in transplanted Paddy crop. This formulation (Fenoxaprop 6\% + Pyrazosulfuron Ethyl 1.5\% + Pretilachlor 50\% ME (ready-mix)) was tried at different concentrations $(800,1000$ and $1200 \mathrm{ml} / \mathrm{ha}$ ). The efficacy of this test herbicide formulation was compared with other herbicide viz., Check I -Chlorimuron Ethyl 25\% WP @ 24g/ ha., Check II-Fenoxaprop-pethyl 9.3\% w/w EC @ 625 ml/ha., Check IIIPretilachlor 50\% EC @ $1500 \mathrm{ml} / \mathrm{ha}$ andCheck IV- Pyrazosulfuron ethyl 10\% WP@150 g/ha. These herbicide treatments were compared with hand weeding, weed free and untreated control treatments.

The herbicide formulation Fenoxaprop 6\%+ Pyrazosulfuron Ethyl 1.5\% + Pretilachlor 50\% ME (ready-mix)was also evaluated for its Phytotoxicity on transplanted Paddy crop in the same experiment in treatments viz., Fenoxaprop 6\% + Pyrazosulfuron Ethyl 1.5\% + Pretilachlor 50\% ME (ready-mix)@ 1000 $\mathrm{ml} / \mathrm{ha}, 2000 \mathrm{ml} / \mathrm{ha}$ and $3000 \mathrm{ml} / \mathrm{ha}$ and were compared with Control.

The experiment was laid out in Randomized Block Design (RBD) with three replications. The plot size was $5.0 \times 3.0 \mathrm{~m}$. A long duration Transplanted Paddy variety Abhilash was used in the study for both Kharif 2018-19and Kharif 2019-20. The seeds were sown in the nursery on $05-07-2018$ during first season and on 08-07-2019 during second season. About twenty five days old seedlings were transplanted in main field on 30-07-2018 during first season and on 02-08-2019 during second season. Spacing adopted was $20 \times 10$ $\mathrm{cm}$. A recommended dose of fertilizer $(75: 75: 87.5 \mathrm{~kg} \mathrm{NPK} / \mathrm{ha})$ was given in threespilt dose. Necessary plant protection and water management practices were followed. The crop was harvested maturity on 15-122018 during first season and on 18-12-2019 during second season to record various yield and yield attributing parameters.

\section{Herbicides application}

The test herbicide viz., Fenoxaprop 6\%+ Pyrazosulfuron Ethyl 1.5\% + Pretilachlor 50\% ME (ready-mix)as well as check herbicide were sprayed on 11-08-2018 during first season and on 15-08-2019 during second season at 1-2 leaf stage of weed flora (12-13 days after transplanting). High volume (Knapsack) sprayer fitted with Flat Fan nozzle was used for spraying. The spray volume used was 500 litres per hectare. The required quantity of herbicide was measured by using pipette after making necessary dilutions and this was mixed in water and used for spraying.

\section{Observations Recorded}

\section{Weed Observations}

\section{Weed Count/Population}

The weed population count (monocot and dicot weeds separately) was recorded at 15,30 and 45 days after application of herbicides. For recording the weed population, an area of $0.5 \times 0.5 \mathrm{~m}\left(0.25 \mathrm{~m}^{2}\right)$ was marked at three spots in each treatment and weed observations were made from the same marked area and average was worked out and expressed in weed number per $\mathrm{m}^{2}$ area. 


\section{Weed Dry Weight}

Weed dry weight of monocot and dicot weeds in different herbicide treatments, were recorded a separate area of $0.5 \times 0.5 \mathrm{~m}$ was marked in each plot and the weeds in each marked quadrant were removed at 15, 30 and 45 days after herbicide spray and oven dried at $80^{\circ} \mathrm{C}$ for 12 hours and weighed to estimate the dry weight of weeds. The weed dry weight was expressed in terms of $\mathrm{g} / \mathrm{m}^{2}$ area.

\section{Weed Control Efficiency}

Weed dry weight recorded at 15, 30 and 45 days after spray of herbicide under absolute control and the respective treatments, the weed control efficiency (WCE) for each treatment was worked out by using formula given below.

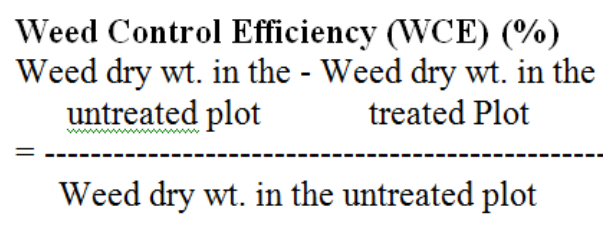

The final weed data (weed dry weight) was transformed using the formula $(\mathrm{X}+0.5)^{0.5}$ for analysis purpose.

\section{Yield and Yield attributes of Transplanted Paddy}

The yield attributes of transplanted Paddy was recorded at harvest of the crop.

\section{Phytotoxicity Observations}

For phytotoxicity evaluation on transplanted Paddy crop, following observations were made by observing temporary or long lasting damage to the leaves if any viz., leaf injury on tips and leaf surface, wilting, vein clearing, necrosis, epinasty and hyponasty at 1, 3, 5, 7 and 10 days after application of test herbicide in the treatments specified for phytotoxicity observations.

\section{Effect on succeeding black gram crop}

To study the effect of Fenoxaprop $6 \%+$ Pyrazosulfuron Ethyl 1.5\% + Pretilachlor 50\% ME (ready-mix)on succeeding black gram was sown in the experimental area previously used for transplanted Paddy crop. The land was prepared manually and blackgram seeds (Var. DU-1) were sown by manually during first and second season, respectively. Recommended practices were followed for both the season. Observations were recorded on germination percentage, phytotoxicity and yield in Black gram.

\section{Statistical Analysis}

The data was analysed in Randomized Complete Block Design (RCBD) by using MSTAC programme. The level of significance used in ' $F$ ' and ' $t$ ' test was $P=0.05$. The critical difference values were calculated wherever the ' $F$ ' test was significant. In other cases values of standard error of means have been provided.

\section{Results and Discussion}

Weed flora after 15, 30 and 45 days after spraying

Both monocot and dicot weeds were observed at 15, 30 and 45 days after spray during both kharif 2018 and kharif 2019 (Table1). Among monocot weeds, Echinochloa crusgalli, Echinochloa colonum, Ludwigia parviflora, Cyperus rotundus, Cyperus difformis, Cyperus iria, Fimbristylis miliacae, Monochoria vaginalis, Leptochloa chinensis and Panicum repens were prominent weeds observed during trail period in experimental plots during both the seasons kharif 2018 and 2019. Ramchandra et al., (2010) and Patra et al., (2011) reported similar weeds in transplanted rice.Raj et al., (2013) reported that in Kuttanad, during kharif season BLW predominate (39.3 per cent) followed by 
sedges (38.9 per cent) and grassy weeds (21.8 per cent) while in rabi season sedges predominate ( 96.8 per cent) followed by BLW ( 2.7 per cent)and grasses ( 0.5 per cent). Singh and Singh (2010) also reported Echinochloa crusgalli, Leptochloa chinensis, Cyperus iria, Fimbristylis miliacea, Ludwigia parviflora, Lindernia crustacea and Monochoriavaginalis as the major weeds of wet seeded rice. Pramanik et al., 2020 also reported complex weed flora: grasses like Echinochloa crusgalli and Panicum repens sedges like Cyperus iria, Cyperus difformis, Fimbristylis miliacae and Cyperus rotundus and broad leaved weeds like Eclipta alba, Ludwigia parviflora, Marsilea quadrifolia and Monochoria vaginalis in transplanted paddy.

\section{Weeddry weight after 15,30 and 45 days after spraying}

All the herbicides reduced the dry weight of grassy weeds, broad-leaf weeds and sedges significantly as compared to the untreated weedy check at 15,30 and 45 days after spraying (DAS) (Table 2). Total monocot weed and dicot dry weight recorded at 15, 30 and 45 DAS showed significant difference among the weed management treatments during kharif 2018. The dry weight of monocot and broad leaved weeds decreased with increase in dose of Fenoxaprop 6\%+ Pyrazosulfuron Ethyl 1.5\% + Pretilachlor 50\% ME (ready-mix) during both the years. In case of 15 DAS lower monocot $\left(0.71 \mathrm{~g} / \mathrm{m}^{2}\right)$ and dicot $\left(0.71 \mathrm{~g} / \mathrm{m}^{2}\right)$ weed dry weight was recorded in weed free plot followed by hand weeding. Among chemical treatments, Fenoxaprop 6\% + Pyrazosulfuron Ethyl 1.5\% + Pretilachlor 50\% ME (ready-mix) @ 1200 $\mathrm{ml} / \mathrm{ha}$ recorded the lowest monocot $\left(3.3 \mathrm{~g} / \mathrm{m}^{2}\right)$ and dicot $\left(1.64 \mathrm{~g} / \mathrm{m}^{2}\right)$ weed dry weight followed by Fenoxaprop 6\% + Pyrazosulfuron Ethyl $1.5 \%$ + Pretilachlor 50\% ME (readymix) @ $1000 \mathrm{ml} / \mathrm{ha}$. The significantly higher total monocot $\left(7.02 \mathrm{~g} / \mathrm{m}^{2}\right)$ and dicot $(3.50$ $\mathrm{g} / \mathrm{m}^{2}$ ) weed dry weight was observed in control plots. Similar trend has also been observed by Muruganand Kathiresan (2010) and Yadav et al., (2009). At 30 DAS, lowest weed dry weight of monocot and dicot weeds were observed from weed free plots followed by Fenoxaprop 6\% + Pyrazosulfuron Ethyl $1.5 \%$ + Pretilachlor 50\% ME (ready-mix) @ 1200, 1000 and $800 \mathrm{ml} / \mathrm{ha}$. Highest weed dry weight was observed in control plots. Similar results were observed at 45 days after application also. The same trend was observed during kharif 2019 also (Table 2). Fenoxaprop- P-ethyl \{ethyl (R)-2-[4-[(6chloro-2-benzoxazolyl) oxy] phenoxy] propanoate $\}$ is an aryloxyphenoxypropionate postemergence herbicide inhibiting fatty acid synthesis in grasses through inhibition of acetyl CoA carboxylase there by controls the weed population and dry weight (Mishra and Pandey,1989).The study conducted by Suryakala et al., (2019) using new herbicides $v i z$. Pretilachlor $6 \%$ + Pyrazosulfuron-ethyl $0.15 \%$ GR @ $10 \mathrm{~kg}$ ha-1 along with post emergence herbicides Fenoxaprop-p-ethyl 9.3\% w/w @ $875 \mathrm{ml}$ ha-1, Bispyribac-sodium 10\% SC @ $200 \mathrm{ml}$ ha-1. Results of the study revealed substantial reduction in weed biomass in all the herbicide treated plots compared to weedy check. Pre-emergence herbicide application followed by application of post emergence herbicides recorded lower weed control efficiency. Due to narrow spectrum of activity, use of single herbicides seldom furnishes satisfactory and season long weed control. The herbicide mixtures (both tank and proprietary mixture) broaden the spectrum of weed control in single application (Damalas, 2005). A grass effective herbicide in combination with a herbicide that kill BLW, would take care of both types; similarly a grass effective herbicide in combination with herbicide that control both BLW and sedges will provide a wider spectrum of weed control (Mukherjee, 2006). Paswan et al., (2012) opined that herbicides with different mode of action when mixed together, bind to different 
target sites in weeds and prevent the probability of target site resistance in susceptible species. Upasani and Barla (2014) reported that application of pyrazosulfuron 25 g ha-3-7DAS being similar to azimsulfuron 50DF $35 \mathrm{~g}$ ha-1 35 DAS and oxyfluorfen 23.5 EC $300 \mathrm{~g}+2,4-\mathrm{D} 80 \% 500 \mathrm{~g}$ ha-1 at 30 and 60 DAS and also fenoxaprop 9.3\% 30DAS $60 \mathrm{~g}$ ha-1 at 60 DAS recorded significantly reduced weed dry matter accumulation by weeds compared to rest of the treatments. Reduced dry matter owing to application of pyrazosulfuron has also been reported by Dixit and Varshney (2008), and Halder et al., (2005).

\section{Weed Control efficiency after 15, 30 and 45 days after spraying}

The data on weed control efficiency against total grassy weed, broad leaved weed and sedge weed were presented in Table (Table 3).During kharif 2018, data indicated that among the chemical weed control treatments, higher weed control efficiency of monocot (78.57 \%) and dicot weeds (81.33) at 15 DAS was recorded in Fenoxaprop 6\%+ Pyrazosulfuron Ethyl 1.5\% + Pretilachlor 50\% ME (ready-mix) @ $1200 \mathrm{ml} / \mathrm{ha}$ followed by Fenoxaprop 6\% + Pyrazosulfuron Ethyl 1.5\% + Pretilachlor 50\% ME (ready-mix) @ 1000 $\mathrm{ml} / \mathrm{ha}$ for different weeds. At 30 days after spraying, maximum weed control efficiency of monocot $(81.25 \%)$ and dicot weeds $(88.34 \%)$ were recorded in Fenoxaprop $6 \%+$ Pyrazosulfuron Ethyl 1.5\% + Pretilachlor 50\% ME (ready-mix) @ 1200 followed by 1000 $\mathrm{ml} / \mathrm{ha}$ only next to weed free. Similarly, at 45 days after spray, Fenoxaprop 6\%+ Pyrazosulfuron Ethyl 1.5\% + Pretilachlor 50\% ME (ready-mix) @ $1200 \mathrm{ml} / \mathrm{ha}$ and 1000 $\mathrm{ml} /$ ha were recorded higher monocot and dicot weed control efficiency. Similar results were observed during kharif 2019 also (Table 3).Increased dosage of new ready mix herbicide recorded increased weed control efficiency at all stages in both the season (Fig
1). Weed control efficiency of Fenoxaprop 6\% + Pyrazosulfuron Ethyl 1.5\% + Pretilachlor $50 \% \mathrm{ME}$ (ready-mix) increased with increase in its dose from $800 \mathrm{ml}$ ha- 1 to $1200 \mathrm{ml}$ ha-1 against total monocot weeds and dicot weeds during first and second year, respectively. Suryakala et al., (2019) with use Preemergence herbicide application followed by application of post emergence herbicides recorded lower weed control efficiency. Weed control index (WCI) ranged from 78.66$92.32 \%$ with various herbicide combinations. Highest WCE (92.32) was recorded in Pretilachlor + Pyrazosulfuron-ethyl + Bispyribac-sodium, while lowest was recorded with twice hand weeding on 20 and 40 DAT (78.66). The results were in conformity with the findings of Porpavai et al., (2006). The highest WCE (97\%) as well as grain yield (6.13Mg ha -1), (Fig 2) was recorded in hand weeded control.

However, this yield was statistically on par with cyhalofop-butyl fs Almix and fenoxaprop p-ethyl fs Almix (5.8 Mg ha-1), which recorded a WCE of 88 and 90 percentage (Prameela et al.,2014). The experiment conducted by Pramanik et al.,(2020) at Agricultural Farm, Institute of Agriculture, Visva-Bharati, West Bengal, results revealed that ready-mix application of fenoxaprop 5\%+ Chlorimuron $0.6 \%+$ Pretilachlor $50 \% \mathrm{ME}$ at 1200 and $1000 \mathrm{ml}$ ha-1 gave higher weed control index of $84.90 \%$ and $81.60 \%$ against grassy weeds, $84.09 \%$ and $82.95 \%$ against broad leaved weeds and $81.89 \%$ and $79.79 \%$ against sedges during 2017 and $84.18 \%$ and $82.95 \%$ against grassy weeds, $80.04 \%$ and $80.84 \%$ against broad leaved weeds and $82.33 \%$ and $79.57 \%$ against sedges.

\section{Effect of different herbicides on yield and yield attributes of Paddy}

During both the season, different weed control treatments had significant influence on yield and yield attributes of paddy(Table 4). Among 
the weed management treatments, weed free check recorded highest number of panicles/hill (21.24) and panicle length $(21.45 \mathrm{~cm})$ followed by Fenoxaprop 6\% + Pyrazosulfuron Ethyl $1.5 \%$ + Pretilachlor $50 \% \mathrm{ME}$ (ready-mix) at $1200 \mathrm{ml} / \mathrm{ha}$ plots which recorded panicle number of 21.08 and panicle length of 21.29 $\mathrm{cm}$ during Kharif 2018-19. Similar results were recorded during kharif 2019 also.

Jayadeva et al., (2009) from Karnataka observed that repeated weeding recorded lower weed dry weight and higher mean grain and straw yield in rice. Whereas, lower grain and straw yield were recorded in weedy check plot.

This is due to the higher infestation of weeds. According to Rajkhowa and Barua (2007) untreated control has recorded significantly lowest yield. The trend was similar with respect to straw yield. Increased grain and straw yield in herbicide treated plots was due to higher weed control efficiency.

Table.1 Crop injury was observed on visual rating from 1-10 scale as given below

\begin{tabular}{|c|c|c|}
\hline Rating & Crop Injury (\%) & Verbal Description \\
\hline $\mathbf{0}$ & - & No symptoms \\
\hline $\mathbf{1}$ & $1-10$ & Very slight discoloration \\
\hline $\mathbf{2}$ & $11-20$ & More severe, but not lasting \\
\hline $\mathbf{3}$ & $21-30$ & Moderate and more lasting \\
\hline $\mathbf{4}$ & $31-40$ & Medium and lasting \\
\hline $\mathbf{5}$ & $41-50$ & Moderately heavy \\
\hline $\mathbf{6}$ & $51-60$ & Heavy \\
\hline $\mathbf{7}$ & $61-70$ & Very Heavy \\
\hline $\mathbf{8}$ & $71-80$ & Nearly destroyed \\
\hline $\mathbf{9}$ & $81-90$ & Destroyed \\
\hline $\mathbf{1 0}$ & $91-100$ & Completely destroyed \\
\hline
\end{tabular}

Table.2 Weed flora observed in the experimental field during kharif 2018 and kharif 2019.

\begin{tabular}{|l|l|l|}
\hline \multicolumn{2}{|c|}{ Monocot weed } & \multicolumn{1}{c|}{ Dicot weed } \\
\hline 1. Echinochloa crusgalli & 6. Cyperus iria & 11. Chenopodium album \\
\hline 2. Echinochloa colonum & 7. Fimbristylis miliacae & 12. Commelina benghalensis \\
\hline 3. Ludwigia parviflora & 8. Monochoria vaginalis & 13. Eclipta alba \\
\hline 4. Cyperus rotundus & 9. Leptochloa chinensis & \\
\hline 5. Cyperus difformis & 10. Panicum repens & \\
\hline
\end{tabular}


Table.3 Weed dry weight $\left(\mathrm{g} / \mathrm{m}^{2}\right)$ recorded in different herbicides treatment in transplanted Paddy.

\begin{tabular}{|c|c|c|c|c|c|c|c|c|c|c|c|c|c|}
\hline \multirow[t]{3}{*}{ Treatments } & \multirow{3}{*}{$\begin{array}{c}\text { Dose } \\
\text { (g/ml/ha) }\end{array}$} & \multicolumn{6}{|c|}{ Kharif 2018} & \multicolumn{6}{|c|}{ Kharif 2019} \\
\hline & & \multicolumn{2}{|c|}{15 DAS } & \multicolumn{2}{|c|}{30 DAS } & \multicolumn{2}{|c|}{45 DAS } & \multicolumn{2}{|c|}{15 DAS } & \multicolumn{2}{|c|}{30 DAS } & \multicolumn{2}{|c|}{45 DAS } \\
\hline & & $\begin{array}{c}\text { Total } \\
\text { Monocot }\end{array}$ & $\begin{array}{l}\text { Total } \\
\text { Dicot }\end{array}$ & $\begin{array}{c}\text { Total } \\
\text { Monocot }\end{array}$ & $\begin{array}{l}\text { Total } \\
\text { Dicot }\end{array}$ & $\begin{array}{c}\text { Total } \\
\text { Monocot }\end{array}$ & $\begin{array}{l}\text { Total } \\
\text { Dicot }\end{array}$ & $\begin{array}{c}\text { Total } \\
\text { Monocot }\end{array}$ & $\begin{array}{l}\text { Total } \\
\text { Dicot }\end{array}$ & $\begin{array}{c}\text { Total } \\
\text { Monocot }\end{array}$ & $\begin{array}{l}\text { Total } \\
\text { Dicot }\end{array}$ & $\begin{array}{c}\text { Total } \\
\text { Monocot }\end{array}$ & $\begin{array}{l}\text { Total } \\
\text { Dicot }\end{array}$ \\
\hline $\mathrm{T}_{1}-\mathrm{NHF}$ & 800 & $\begin{array}{c}3.73 \\
(13.39)\end{array}$ & $\begin{array}{c}1.93 \\
(3.23)\end{array}$ & $\begin{array}{c}7.11 \\
(50.09)\end{array}$ & $\begin{array}{c}3.30 \\
(10.36)\end{array}$ & $\begin{array}{c}12.07 \\
(145.22)\end{array}$ & $\begin{array}{c}5.39 \\
(28.60)\end{array}$ & $\begin{array}{c}4.12 \\
(16.50)\end{array}$ & $\begin{array}{c}2.11 \\
(3.97)\end{array}$ & $\begin{array}{c}7.89 \\
(61.68)\end{array}$ & $\begin{array}{c}3.64 \\
(12.71)\end{array}$ & $\begin{array}{c}13.38 \\
(178.54)\end{array}$ & $\begin{array}{c}5.97 \\
(35.10)\end{array}$ \\
\hline $\mathrm{T}_{2}-\mathrm{NHF}$ & 1000 & $\begin{array}{c}3.43 \\
(11.25)\end{array}$ & $\begin{array}{c}1.72 \\
(2.46)\end{array}$ & $\begin{array}{c}6.56 \\
(42.51)\end{array}$ & $\begin{array}{c}2.90 \\
(7.93)\end{array}$ & $\begin{array}{c}11.00 \\
(120.45)\end{array}$ & $\begin{array}{c}4.68 \\
(21.39)\end{array}$ & $\begin{array}{c}3.79 \\
(13.84)\end{array}$ & $\begin{array}{c}1.88 \\
(3.03\end{array}$ & $\begin{array}{c}7.27 \\
(52.32)\end{array}$ & $\begin{array}{c}3.21 \\
(9.80)\end{array}$ & $\begin{array}{c}12.19 \\
(148.22)\end{array}$ & $\begin{array}{c}5.18 \\
(26.35)\end{array}$ \\
\hline $\mathrm{T}_{3}-\mathrm{NHF}$ & 1200 & $\begin{array}{c}3.31 \\
(10.45)\end{array}$ & $\begin{array}{c}1.64 \\
(2.19)\end{array}$ & $\begin{array}{c}6.24 \\
(38.44)\end{array}$ & $\begin{array}{c}2.72 \\
(6.90)\end{array}$ & $\begin{array}{c}10.47 \\
(109.12)\end{array}$ & $\begin{array}{c}4.36 \\
(18.49)\end{array}$ & $\begin{array}{c}3.66 \\
(12.88)\end{array}$ & $\begin{array}{c}1.79 \\
(2.72)\end{array}$ & $\begin{array}{c}6.91 \\
(47.26)\end{array}$ & $\begin{array}{c}2.99 \\
(8.42)\end{array}$ & $\begin{array}{c}11.61 \\
(134.36)\end{array}$ & $\begin{array}{c}4.83 \\
(22.80)\end{array}$ \\
\hline $\mathbf{T}_{4}$-Check I & 24 & $\begin{array}{c}4.96 \\
(24.07)\end{array}$ & $\begin{array}{c}2.47 \\
(5.60)\end{array}$ & $\begin{array}{c}9.64 \\
(92.48)\end{array}$ & $\begin{array}{c}4.41 \\
(18.96)\end{array}$ & $\begin{array}{c}15.76 \\
(247.82)\end{array}$ & $\begin{array}{c}7.05 \\
(49.19)\end{array}$ & $\begin{array}{c}5.48 \\
(29.57)\end{array}$ & $\begin{array}{c}2.71 \\
(6.85)\end{array}$ & $\begin{array}{c}10.68 \\
(113.67)\end{array}$ & $\begin{array}{c}4.88 \\
(23.27)\end{array}$ & $\begin{array}{c}17.48 \\
(304.88)\end{array}$ & $\begin{array}{c}7.81 \\
(60.47)\end{array}$ \\
\hline $\mathbf{T}_{5}$-Check II & 625 & $\begin{array}{c}3.91 \\
(14.78)\end{array}$ & $\begin{array}{c}2.31 \\
(4.82)\end{array}$ & $\begin{array}{c}7.55 \\
(56.51)\end{array}$ & $\begin{array}{c}4.04 \\
(15.80)\end{array}$ & $\begin{array}{c}13.04 \\
(169.51)\end{array}$ & $\begin{array}{c}6.75 \\
(45.03)\end{array}$ & $\begin{array}{c}4.32 \\
(18.17)\end{array}$ & $\begin{array}{c}2.54 \\
(5.95)\end{array}$ & $\begin{array}{c}8.37 \\
(69.53)\end{array}$ & $\begin{array}{c}4.47 \\
(19.47)\end{array}$ & $\begin{array}{c}14.45 \\
(208.30)\end{array}$ & $\begin{array}{c}7.47 \\
(55.30)\end{array}$ \\
\hline $\begin{array}{c}\mathbf{T}_{6}-\text { Check } \\
\text { III }\end{array}$ & 1500 & $\begin{array}{c}4.30 \\
(17.97)\end{array}$ & $\begin{array}{c}2.30 \\
(4.80)\end{array}$ & $\begin{array}{c}8.43 \\
(70.61)\end{array}$ & $\begin{array}{c}4.09 \\
(16.24)\end{array}$ & $\begin{array}{c}14.14 \\
(199.58)\end{array}$ & $\begin{array}{c}6.68 \\
(44.18)\end{array}$ & $\begin{array}{c}4.75 \\
(22.10)\end{array}$ & $\begin{array}{c}2.53 \\
(5.92)\end{array}$ & $\begin{array}{c}9.35 \\
(86.92)\end{array}$ & $\begin{array}{c}4.52 \\
(19.95)\end{array}$ & $\begin{array}{c}15.68 \\
(245.47)\end{array}$ & $\begin{array}{c}7.41 \\
(54.40)\end{array}$ \\
\hline $\begin{array}{c}\text { T }_{7} \text {-Check } \\
\text { IV }\end{array}$ & 150 & $\begin{array}{c}4.55 \\
(20.16)\end{array}$ & $\begin{array}{c}2.27 \\
(4.65)\end{array}$ & $\begin{array}{c}8.86 \\
(78.04)\end{array}$ & $\begin{array}{c}4.00 \\
(15.53)\end{array}$ & $\begin{array}{c}14.67 \\
(214.58)\end{array}$ & $\begin{array}{c}6.46 \\
(41.22)\end{array}$ & $\begin{array}{c}5.03 \\
(24.78)\end{array}$ & $\begin{array}{c}2.49 \\
(5.71)\end{array}$ & $\begin{array}{c}9.83 \\
(96.06)\end{array}$ & $\begin{array}{c}4.43 \\
(19.14)\end{array}$ & $\begin{array}{c}16.26 \\
(263.97)\end{array}$ & $\begin{array}{c}7.16 \\
(50.75)\end{array}$ \\
\hline $\begin{array}{c}\text { T8-Hand } \\
\text { Weeding } \\
\text { @ } 15 \text { \& } 30\end{array}$ & - & $\begin{array}{c}3.60 \\
(12.43)\end{array}$ & $\begin{array}{c}1.78 \\
(2.68)\end{array}$ & $\begin{array}{c}7.02 \\
(48.81)\end{array}$ & $\begin{array}{c}3.10 \\
(9.13)\end{array}$ & $\begin{array}{c}11.90 \\
(141.09)\end{array}$ & $\begin{array}{c}4.72 \\
(21.80)\end{array}$ & $\begin{array}{c}3.97 \\
(15.28)\end{array}$ & $\begin{array}{c}1.69 \\
(2.36)\end{array}$ & $\begin{array}{c}7.78 \\
(60.09)\end{array}$ & $\begin{array}{c}3.43 \\
(11.28)\end{array}$ & $\begin{array}{c}12.17 \\
(147.73)\end{array}$ & $\begin{array}{c}5.37 \\
(28.29)\end{array}$ \\
\hline $\begin{array}{c}T_{9} \text {-Weed } \\
\text { free }\end{array}$ & - & $\begin{array}{c}0.71 \\
(0.00)\end{array}$ & $\begin{array}{c}0.71 \\
(0.00)\end{array}$ & $\begin{array}{c}0.71 \\
(0.00)\end{array}$ & $\begin{array}{c}0.71 \\
(0.00)\end{array}$ & $\begin{array}{c}0.71 \\
(0.00)\end{array}$ & $\begin{array}{c}0.71 \\
(0.00)\end{array}$ & $\begin{array}{c}0.71 \\
(0.00)\end{array}$ & $\begin{array}{c}0.71 \\
(0.00)\end{array}$ & $\begin{array}{c}0.71 \\
(0.00)\end{array}$ & $\begin{array}{c}0.71 \\
(0.00)\end{array}$ & $\begin{array}{c}0.71 \\
(0.00)\end{array}$ & $\begin{array}{c}0.71 \\
(0.00)\end{array}$ \\
\hline $\mathbf{T}_{10}$-Control & - & $\begin{array}{c}7.02 \\
(48.78)\end{array}$ & $\begin{array}{c}3.50 \\
(11.73)\end{array}$ & $\begin{array}{c}14.34 \\
(205.00) \\
\end{array}$ & $\begin{array}{c}7.73 \\
(59.20) \\
\end{array}$ & $\begin{array}{c}26.66 \\
(710.43)\end{array}$ & $\begin{array}{c}12.46 \\
(154.72)\end{array}$ & $\begin{array}{c}7.96 \\
(62.81)\end{array}$ & $\begin{array}{c}3.95 \\
(15.13)\end{array}$ & $\begin{array}{c}14.75 \\
(217.20)\end{array}$ & $\begin{array}{c}7.42 \\
(54.62)\end{array}$ & $\begin{array}{c}24.99 \\
(624.00) \\
\end{array}$ & $\begin{array}{c}12.66 \\
(159.78)\end{array}$ \\
\hline SE(m) & - & 1.061 & 0.258 & 4.445 & 1.288 & 15.024 & 3.298 & 1.361 & 0.330 & 4.766 & 1.193 & 13.142 & 3.399 \\
\hline C.D. & - & 3.176 & 0.772 & 13.309 & 3.856 & 44.984 & 9.876 & 4.076 & 0.988 & 14.269 & 3.572 & 39.350 & 10.176 \\
\hline
\end{tabular}

\# Figures in parenthesis are Actual values. DAS-Days After Spray

NHF - New Herbicidal Formulation : Fenoxaprop 6\% + Pyrazosulfuron Ethyl 1.5\% + Pretilachlor 50\% ME (ready-mix) 
Table.4 Weed control efficiency (\%) recorded in different herbicides treatment recorded in transplanted Paddy.

\begin{tabular}{|c|c|c|c|c|c|c|c|c|c|c|c|c|c|}
\hline \multirow[t]{3}{*}{ Treatments } & \multirow{3}{*}{$\begin{array}{c}\text { Dose } \\
\text { (g/ml/ha) }\end{array}$} & \multicolumn{6}{|c|}{ Kharif 2018} & \multicolumn{6}{|c|}{ Kharif 2019} \\
\hline & & \multicolumn{2}{|c|}{15 DAS } & \multicolumn{2}{|c|}{30 DAS } & \multicolumn{2}{|c|}{45 DAS } & \multicolumn{2}{|c|}{15 DAS } & \multicolumn{2}{|c|}{30 DAS } & \multicolumn{2}{|c|}{45 DAS } \\
\hline & & $\begin{array}{c}\text { Total } \\
\text { Monocot }\end{array}$ & $\begin{array}{l}\text { Total } \\
\text { Dicot }\end{array}$ & $\begin{array}{c}\text { Total } \\
\text { Monocot }\end{array}$ & $\begin{array}{l}\text { Total } \\
\text { Dicot }\end{array}$ & $\begin{array}{c}\text { Total } \\
\text { Monocot }\end{array}$ & $\begin{array}{l}\text { Total } \\
\text { Dicot }\end{array}$ & $\begin{array}{c}\text { Total } \\
\text { Monoc } \\
\text { ot }\end{array}$ & $\begin{array}{l}\text { Total } \\
\text { Dicot }\end{array}$ & $\begin{array}{c}\text { Total } \\
\text { Monoc } \\
\text { ot }\end{array}$ & $\begin{array}{l}\text { Total } \\
\text { Dicot }\end{array}$ & $\begin{array}{c}\text { Total } \\
\text { Monoc } \\
\text { ot }\end{array}$ & $\begin{array}{l}\text { Total } \\
\text { Dicot }\end{array}$ \\
\hline $\mathrm{T}_{1}$-NHF & 800 & 72.55 & 72.49 & 75.57 & 82.50 & 79.56 & 81.52 & 73.73 & 73.79 & 71.60 & 76.72 & 71.39 & 78.03 \\
\hline $\mathrm{T}_{2}$-NHF & 1000 & 76.93 & 79.07 & 79.27 & 86.61 & 83.05 & 86.18 & 77.97 & 80.00 & 75.91 & 82.07 & 76.25 & 83.51 \\
\hline $\mathbf{T}_{3}$-NHF & 1200 & 78.57 & 81.33 & 81.25 & 88.34 & 84.64 & 88.05 & 79.49 & 82.02 & 78.24 & 84.58 & 78.47 & 85.73 \\
\hline $\mathbf{T}_{4}$-Check I & 24 & 50.67 & 52.27 & 54.89 & 67.97 & 65.12 & 68.21 & 52.92 & 54.73 & 47.67 & 57.40 & 51.14 & 62.15 \\
\hline $\mathbf{T}_{5}$-Check II & 625 & 69.71 & 58.90 & 72.43 & 73.31 & 76.14 & 70.90 & 71.07 & 60.67 & 67.99 & 64.36 & 66.62 & 65.39 \\
\hline $\mathbf{T}_{6}$-Check III & 1500 & 63.17 & 59.09 & 65.56 & 72.57 & 71.91 & 71.45 & 64.81 & 60.88 & 59.98 & 63.47 & 60.66 & 65.95 \\
\hline $\mathrm{T}_{7}$-Check IV & 150 & 58.67 & 60.41 & 61.93 & 73.77 & 69.80 & 73.36 & 60.54 & 62.24 & 55.77 & 64.97 & 57.70 & 68.23 \\
\hline $\begin{array}{l}\text { T8-Hand } \\
\text { Weeding } \\
\text { @ } 15 \text { \& } 30\end{array}$ & - & 74.53 & 77.15 & 76.19 & 84.58 & 80.14 & 85.91 & 75.67 & 84.42 & 72.34 & 79.36 & 76.33 & 82.29 \\
\hline $\mathbf{T}_{9}$-Weed free & - & 100.00 & 100.00 & 100.00 & $\begin{array}{c}100.0 \\
0\end{array}$ & 100.00 & 100.00 & 100.00 & $\begin{array}{c}100.0 \\
0\end{array}$ & 100.00 & 100.00 & 100.00 & 100.00 \\
\hline $\mathbf{T}_{10}$-Control & - & -- & -- & -- & -- & -- & -- & -- & -- & -- & -- & -- & -- \\
\hline
\end{tabular}

*DAS-Days After Spray, NHF - New Herbicidal Formulation : Fenoxaprop 6\% + Pyrazosulfuron Ethyl 1.5\% + Pretilachlor 50\% ME (ready-mix) 
Table.5 Effect of different herbicides on yield and yield attributes of Paddy

\begin{tabular}{|c|c|c|c|c|c|c|c|}
\hline \multirow[t]{2}{*}{ Treatments } & \multirow{2}{*}{$\begin{array}{c}\text { Dose } \\
\text { (g/ml/ha) }\end{array}$} & \multicolumn{3}{|c|}{ Kharif-2018 } & \multicolumn{3}{|c|}{ Kharif-2019 } \\
\hline & & $\begin{array}{c}\text { No. of } \\
\text { Panicles/hill }\end{array}$ & $\begin{array}{c}\text { Panicle } \\
\text { length }(\mathrm{cm})\end{array}$ & $\begin{array}{c}\text { Grain yield } \\
\text { (kg/ha) }\end{array}$ & $\begin{array}{c}\text { No. of } \\
\text { Panicles/hill }\end{array}$ & $\begin{array}{c}\text { Panicle } \\
\text { length }(\mathrm{cm})\end{array}$ & $\begin{array}{c}\text { Grain yield } \\
\text { (kg/ha) }\end{array}$ \\
\hline $\mathrm{T}_{1}$-NHF & 800 & 20.33 & 20.53 & 4278 & 19.72 & 19.91 & 4321 \\
\hline $\mathrm{T}_{2}-\mathrm{NHF}$ & 1000 & 20.73 & 20.93 & 4463 & 20.11 & 20.31 & 4506 \\
\hline $\mathbf{T}_{3}-\mathrm{NHF}$ & 1200 & 21.08 & 21.29 & 4481 & 20.45 & 20.65 & 4525 \\
\hline $\mathbf{T}_{4}$-Check I & 24 & 19.90 & 20.11 & 3990 & 19.30 & 19.50 & 4032 \\
\hline $\mathbf{T}_{5}$-Check II & 625 & 19.84 & 20.04 & 4078 & 19.24 & 19.43 & 4119 \\
\hline $\mathbf{T}_{6}$-Check III & 1500 & 20.30 & 20.50 & 4001 & 19.69 & 19.88 & 4142 \\
\hline $\mathbf{T}_{7}$-Check IV & 150 & 20.18 & 20.38 & 4121 & 19.57 & 19.77 & 4263 \\
\hline $\begin{array}{c}\text { T8-Hand } \\
\text { Weeding } \\
\text { @ } 15 \text { \& } 30\end{array}$ & - & 20.58 & 20.78 & 3862 & 19.96 & 20.16 & 3904 \\
\hline$T_{9}$-Weed free & - & 21.24 & 21.45 & 4511 & 20.63 & 20.84 & 4554 \\
\hline $\mathbf{T}_{10}$-Control & - & 19.40 & 19.59 & 3276 & 18.82 & 19.00 & 3313 \\
\hline SE(m) & - & $\mathbf{0 . 0 3 7}$ & 0.036 & 27.492 & 0.037 & 0.037 & 27.593 \\
\hline C.D. & - & 0.111 & 0.108 & 82.315 & 0.110 & 0.111 & 82.617 \\
\hline
\end{tabular}

NHF - New Herbicidal Formulation : Fenoxaprop 6\% + Pyrazosulfuron Ethyl 1.5\% + Pretilachlor 50\% ME (ready-mix) 
Table.6 Phytotoxicity studies on transplanted paddy during both Kharif 2018 and Kharif 2019.

\begin{tabular}{|c|c|c|c|c|c|c|}
\hline Treatments & $\begin{array}{c}\text { Hyponast } \\
\mathbf{y}\end{array}$ & $\begin{array}{c}\text { Epinast } \\
\mathbf{y}\end{array}$ & $\begin{array}{c}\text { Leaf } \\
\text { yellowing }\end{array}$ & $\begin{array}{c}\text { Leaf } \\
\text { scorchin } \\
\mathbf{g}\end{array}$ & $\begin{array}{c}\text { Leaf } \\
\text { Drying }\end{array}$ & $\begin{array}{c}\text { Leaf } \\
\text { mortal } \\
\text { ity }\end{array}$ \\
\hline $\mathbf{T}_{\mathbf{1}}$ - NHF @ $\mathbf{1 0 0 0} \mathbf{~ m l / h a}$ & 0 & 0 & 0 & 0 & 0 & $\mathbf{0}$ \\
\hline $\mathbf{T}_{\mathbf{2}}$ - NHF@ $\mathbf{2 0 0 0} \mathbf{~ m l} / \mathbf{h a}$ & 0 & 0 & 0 & 0 & 0 & $\mathbf{0}$ \\
\hline $\mathbf{T}_{\mathbf{3}}$ - NHF @ $\mathbf{3 0 0 0} \mathbf{~ m l / h a}$ & 0 & 0 & 0 & 0 & 0 & $\mathbf{0}$ \\
\hline Control & $\mathbf{0}$ & $\mathbf{0}$ & $\mathbf{0}$ & $\mathbf{0}$ & $\mathbf{0}$ & $\mathbf{0}$ \\
\hline 0 - No Phytotoxicity & 10- Complete kill of the plant \\
NHF - New Herbicidal Formulation : Fenoxaprop 6\% + Pyrazosulfuron Ethyl 1.5\% + Pretilachlor 50\% ME (ready-mix)
\end{tabular}

Table.7 Effect of different herbicides on germination per cent of black gram

\begin{tabular}{|c|c|c|c|c|c|}
\hline \multirow[t]{2}{*}{ Treatment } & \multirow{2}{*}{$\begin{array}{c}\text { Dose } \\
\text { (g/ml/ha) }\end{array}$} & \multicolumn{2}{|c|}{ Kharif - 2018-19 } & \multicolumn{2}{|c|}{ Kharif - 2019-20 } \\
\hline & & $\begin{array}{c}\text { Germination } \\
\text { per cent }\end{array}$ & $\begin{array}{c}\text { Black gram } \\
\text { Grain Yield } \\
\quad \text { (kg/ha) }\end{array}$ & $\begin{array}{c}\text { Germination } \\
\text { per cent }\end{array}$ & $\begin{array}{c}\text { Black gram } \\
\text { Grain Yield } \\
\text { (kg/ha) }\end{array}$ \\
\hline $\mathrm{T}_{1}$-NHF & 800 & 87.92 & 579.53 & 89.68 & 591.12 \\
\hline $\mathbf{T}_{2}$-NHF & 1000 & 88.72 & 593.64 & 90.50 & 605.51 \\
\hline $\mathbf{T}_{3}-\mathrm{NHF}$ & 1200 & 90.01 & 618.11 & 91.81 & 630.47 \\
\hline $\mathbf{T}_{4}$-Check I & 24 & 88.83 & 580.47 & 90.60 & 592.08 \\
\hline $\mathbf{T}_{5}$-Check II & 625 & 87.82 & 576.71 & 89.57 & 588.24 \\
\hline $\mathrm{T}_{6}$-Check III & 1500 & 88.45 & 588.00 & 90.22 & 599.76 \\
\hline T $_{7}$-Check IV & 150 & 88.72 & 573.89 & 90.50 & 585.37 \\
\hline $\begin{array}{c}\mathbf{T}_{8} \text {-Hand Weeding @ } 15 \\
\text { \& } 30\end{array}$ & - & 89.36 & 608.70 & 91.15 & 620.87 \\
\hline$T_{9}$-Weed free & - & 89.63 & 594.59 & 91.42 & 606.48 \\
\hline $\mathbf{T}_{10}$-Control & - & 89.19 & 581.41 & 90.97 & 593.04 \\
\hline SEm \pm & - & - & 1.110 & - & 1.113 \\
\hline CD & - & - & 3.324 & - & 3.334 \\
\hline
\end{tabular}

NHF - New Herbicidal Formulation : Fenoxaprop 6\% + Pyrazosulfuron Ethyl 1.5\% + Pretilachlor 50\% ME (ready-mix) 
Fig.1

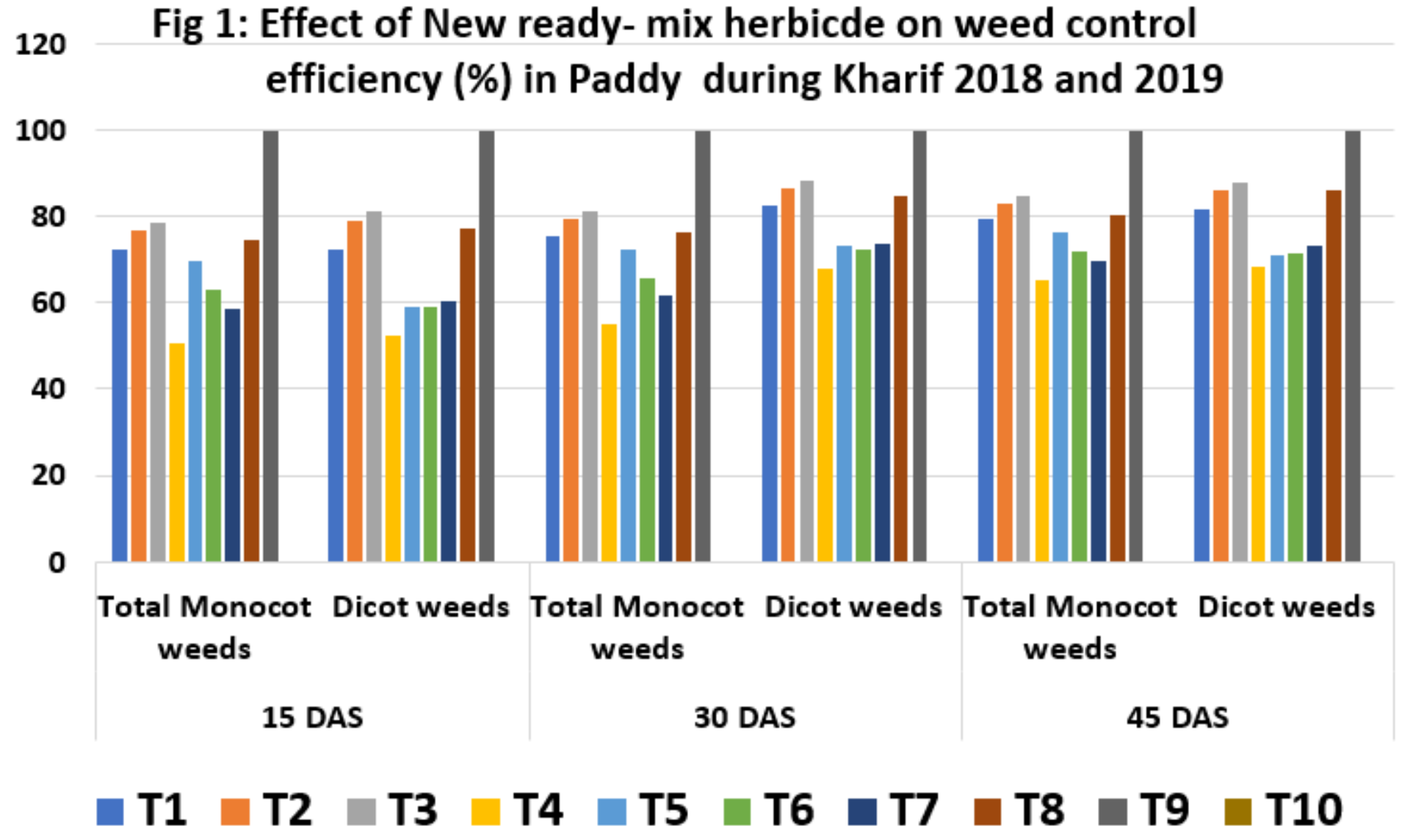


Fig.2

Fig 2: Effect of New Ready-Mix herbicide on Grain yield of Paddy during 2018 and 2019

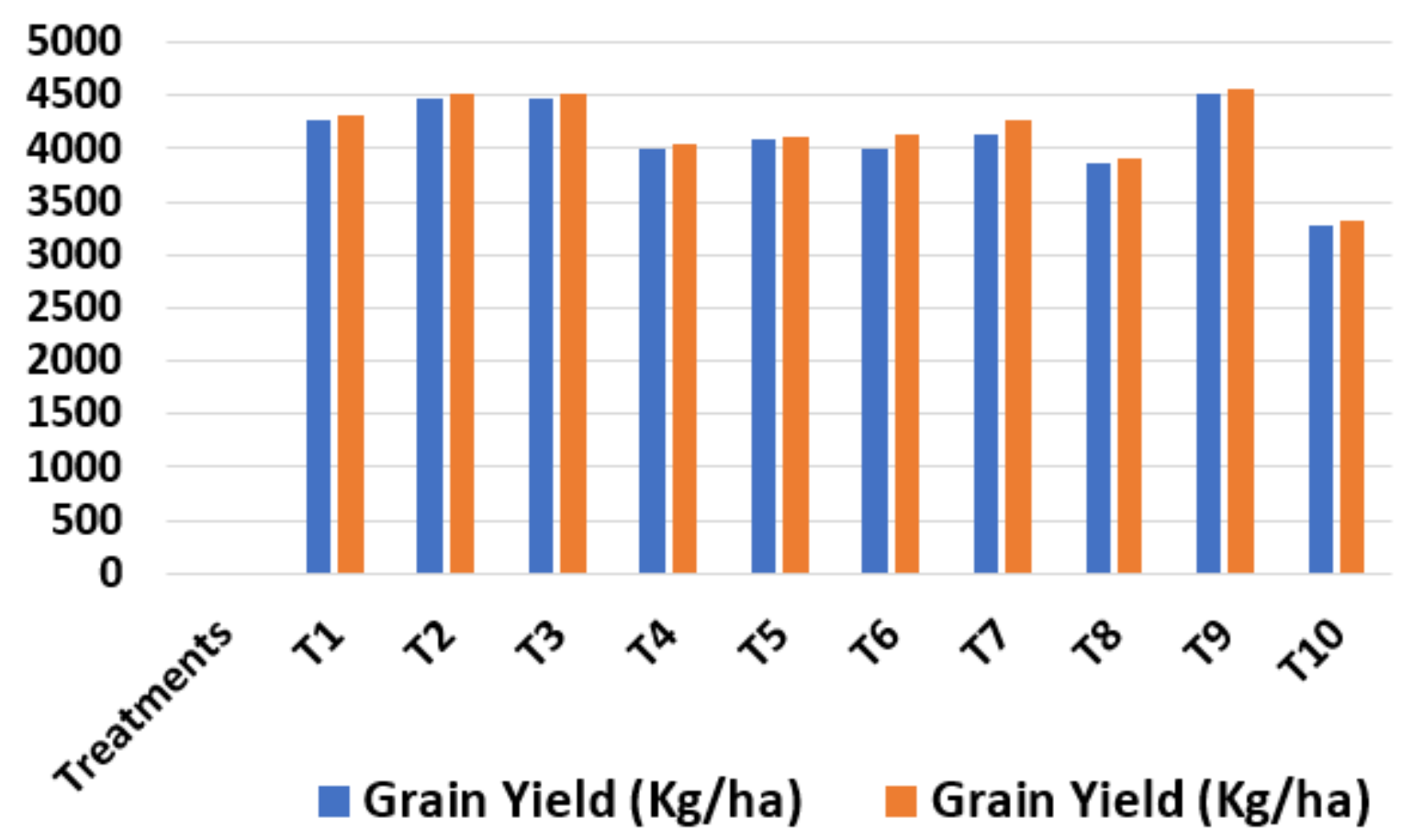


Nadeem Akbar et al., (2011) reported higher weed suppression and increase in rice yield by $25 \%$ over control. Reddy et al., (2003) from Hyderabad noticed that Cyperus spp., Paspalum spp., Caesulia axillaris, Rotala densiflora and Monocharia vaginalis caused $28-40 \%$ reduction in yield of transplanted rice. Application of Fenoxaprop $6 \%+$ Pyrazosulfuron Ethyl 1.5\% + Pretilachlor 50\% ME (ready-mix) at $1200 \mathrm{ml} / \mathrm{ha}$ and weed free check recorded significantly superior yield over rest of the herbicidal treatments and weedy check.

Among the weed management treatments, weed free check recorded highest grain yield (4511 kg/ha \& $4554 \mathrm{~kg} / \mathrm{ha}$ ) followed by Fenoxaprop 6\% + Pyrazosulfuron Ethyl 1.5\% + Pretilachlor 50\% ME (ready-mix) at 1200 $\mathrm{ml} / \mathrm{ha}$ plots which recorded grain yield of $4481 \mathrm{~kg} / \mathrm{ha} \& 4525 \mathrm{~kg} / \mathrm{ha} \& 1000 \mathrm{ml} / \mathrm{ha}(4463$ $\mathrm{kg} / \mathrm{ha} \& 4506 \mathrm{~kg} / \mathrm{ha}$ ) during Kharif 2018-19 \& Kharif 2019-20,respectively.The data of trial conducted by Suryakala et al., (2019a) revealed that significantly higher grain yield and straw yield were recorded with Pretilachlor + Pyrazosulfuron-ethyl + Bispyribac-sodium (5163 and $7654 \mathrm{~kg}$ ha-1) followed by Pretilachlor + Pyrazosulfuronethyl + Fenoxaprop-p-ethyl(4965 and7366 kg ha-1) and was at par with twice hand weeding on 20 and 40 DAT (4787 and $7150 \mathrm{~kg}$ ha-1), respectively. The lowest grain and straw yield (3046 and $4600 \mathrm{~kg}$ ha-1) were recorded with un weeded control, respectively indicating the importance of weed management in the critical growth period of crop by herbicide application, which facilitated the efficient use of resources. The findings of these investigations were in line with Vivek Yadav and Bhagwan Singh, (2006).Pramanik et al.,(2020)reported that application of Fenoxaprop+Chlorimuron+Pretilachlor 1200 $\mathrm{ml}$ ha-1and $1000 \mathrm{ml}$ ha-1 resulted into the higher number of panicles $\mathrm{m}-2$, test weight and grain yield (6088 and $6058 \mathrm{~kg}$ ha- 1 in 2017 and 6513 and 6490 kgha-1 in 2018).
Both were superior to its lower doses $(800 \mathrm{ml}$ ha-1) and chlorimuron ethyl, fenoxaprop-pethyl, pretilachlor and pyrazosulfuron ethyl during both the years. Suryakala et al., (2019b) reported that pre-emergence herbicide application of Pretilachlor + Pyrazosulfuronethyl followed by post emergence herbicide application of Bispyribac-sodium recorded lower weed population, higher yield parameters, grain yield (5163 kg ha- 1) and straw yield (7654 kg ha-1) followed by Pretilachlor + Pyrazosulfuron-ethyl + Fenoxaprop-p-ethyl. There was increased yield with increase in dosage of new ready mix herbicide in the both the year (Fig 2).

\section{Phytotoxic effect of herbicides on transplanted paddy crop.}

The effect of phytotoxicity of herbicides on transplanted paddy crop (average of 1-10 days observation is presented in table5. No Visual symptoms of injury or phytotoxicity of Fenoxaprop 6\% + Pyrazosulfuron Ethyl 1.5\% + Pretilachlor 50\% ME (ready-mix)were observed in even higher concentration of 3000 $\mathrm{ml} / \mathrm{ha}$ from one day application to 10 days after application during both the seasons (Table 5). Similar results were reported by many researchers viz., Pramanik et al.,(2020); Yadav et al., 2019 and Pal et al., (2002)

\section{Effect of herbicides on succeeding blackgram.}

\section{Germination per cent of black gram}

The germination of succeeding blackgram was not affected by Fenoxaprop 6\%+ Pyrazosulfuron Ethyl 1.5\% + Pretilachlor 50\% ME (ready-mix) applied at different doses to previous crop of transplanted paddy for the purpose of weed control in transplanted paddy. The germination per cent in herbicide applied plots were recorded on par with weed free check and control plot (Ranges from 87.92 to $91.81 \%$ ) during both the season 
(Table 6). This results indicated that, application of Fenoxaprop $6 \%+$ Pyrazosulfuron Ethyl 1.5\% + Pretilachlor 50\% ME (ready-mix) applied at different doses to previous crop of transplanted paddy for the purpose of weed control in transplanted paddy was not affected the succeeding crop of black gram.

\section{Yield of Black gram}

The grain yield of succeeding black gram was also not differed significantly due to different herbicidal treatments indicating no adverse effect of Fenoxaprop $6 \%+$ Pyrazosulfuron Ethyl $1.5 \%$ + Pretilachlor 50\% ME (readymix)on succeeding crop of black gram during both the season (Table 6). Yield of black gram showed that, application of Fenoxaprop 6\%+ Pyrazosulfuron Ethyl 1.5\% + Pretilachlor 50\% ME (ready-mix) applied at different doses to previous crop of transplanted paddy for the purpose of weed control in transplanted paddy was safe to succeeding crop of black gram.

Based on two season trials, application of Fenoxaprop 6\% + Pyrazosulfuron Ethyl 1.5\% + Pretilachlor 50\% ME (ready-mix)@ all the doses showed good control on different weed flora and accordingly higher grain yield was observed. Fenoxaprop 6\% + Pyrazosulfuron Ethyl $1.5 \%$ + Pretilachlor 50\% ME (readymix)@1000-1200 ml/ha showed best results to other treatments therefore it can be recommended for control of major weeds in transplanted paddy.

\section{Acknowledgement}

The author is thankful to $\mathrm{M} / \mathrm{s}$ Willowood Chemicals Private Limited, New Delhi for providing financial assistant to UAS, Dharwad for conducting the experiment.

\section{References}

Alstorm, S. Fundamental of Weed Management in Hot Climatic Peasant
Agriculture. Swedish University of Agric. Sci., Uppsala, Sweden, 1990, pp. 50-53.

Avudaithai, S. and Veerabadran, V. Effect of herbicide mixtures on the total weed spectrum in transplanted rice. Crop Res, 2000, 19: 6-12.

Balasubramanian, P. and Palaniappan S. P., Principles and practices of Agronomy. Agrobios Publishing co. Pvt. Ltd., New Delhi,2001: 306-364.

Barret, S. C. H. and Seaman, D. E. The weed flora of Californian rice fields. Aquatic Bot., 1980, 9: 351-376.

Damalas, C. A. Herbicide tank mixture: common interactions. Int. J. Agric. Biol. 2005, 6: 209-212.

Dixit, A. and Varshney, J. G. 2008. Assessment of post emergence herbicides in direct seeded rice. Indian J. Weed Sci.,2008, 40: 144-147.

Duary, B., Mishra, M. M., Dash, R. and Teja, K. C. (2015). Weed management in lowland rice. Indian J. Weed Sci.,2015, 47:224-232.

Halder, P., Maiti, S., Bhattacharya, S. P. and Banerjee, H. Comparative efficacy of Pyrazosulfuron Ethyl (PSE) alone andits combination with Molinate against weed complex of boropaddy. $J$. Crop Weed,2005, 1 : 49-53.

Jayadeva, H. M., Bhairappanavar, S. T., Somashekharappa, P.R. and Rangaswamy, B.R. Efficacy of azimsulfuron for weed control in transplanted rice. Indian $\mathrm{J}$ of Weed Sci., 2009;41(3\&4):172-175.

Joy, P. P., Syriac, E. K., Nair, P. K. C. and Joseph, C. A. Weed control in wet seeded rice in Kerala, India. IRRI Newsl., 1991, 16(6):25.

Juraimi, A. S., Saiful, A. H. M., Uddin, M. K., Anwar M. P., Azmi M., Sustainable weed management in direct seeded rice culture: A review. Aust. J. Crop Sci.,2013, 7(7):989-1002. 
Mishra, A. K and Pandey, A. B. Toxicity of three herbicides to some nitrogenfixing cyanobacteria. Ecotoxicol Environ Saf., 1989, 17 (2): 236-46.

Mukherjee, D. Weed management strategy in rice-a review. Agric. Rev.2006, 27: 247-257.

Murugan, G. and Kathiresan, R. M. A survey of weed flora in rice fields of Cuddalore district of Tamil Nadu, India. Plant archives. 2010; 10(2):647649.

Nadeem Akbar, Eh Sanulla, KhawarJabran and Mohammad Amjad Ali. Weed management improves yield and quality of direct seeded rice. Aus. $J$ Crop Sci., 2011, 5(6):688-694.

Pal, D., Ghosh, R. K. and Bhowmick, M. K. Bio efficacy of new promicing herbicides for weed management in summer rice. Indian Rice J.Weed Sci., 2002,32(1/2):35-38

Paswan, A., Kumar, R., Kumar, P., and Singh, R. K. (2012). Influence of Metsulfuron-methyl and carfentrazoneethyleither alone or in combination on weed flora, crop growth and yield in wheat (Triticum aestivum). Madras Agric. J. 99: 560-562.

Patra A.K., Halder, J. and Mishra, M.M. Chemical weed control in transplanted rice in Hirakud command area of Orissa. Indian J of Weed Sci. 2011, 43(3\&4):175-177.

Porpavai, A., Anbumani, S. and Jayraj, T. Integrated weed management in drum seeded rice. Agric. Sci. Digest. 2006, 26(4):294-296.

Pramanik, K., Shah, M. H., Rahul Kumar Gupta, R.K. and Singhal, M. Bioefficacy of Ready-mix Herbicide on Weed Flora and Productivity of Transplanted Rice. International Journal of Bio-resource and Stress Management, 2020,11(2):132-137.

Prameela, P., Menon, S. S., Meera V Menon,
M. V., Effect of new post emergence herbicides on weed dynamics in wet seeded Rice. Journal of Tropical Agriculture, 2014, 52 (1) : 94 -100, 2014.

Rajkhowa, D. J and Barua, I. C. Integrated weed management in upland rice in Assam. Indian J Weed Sci., 2007; 39(3\&4):176-177.

Raj, S. K., Mathew, R., Jose, N. and Leenakumary, S. Evaluation of early post emergence and post emergence herbicides on weed control and productivity of direct-seeded puddled rice in Kuttanad. Madras. Agric. J., 2013, 100:738-742.

Ramchandra, C., Denesh, G. R. and Sydanwarulla. Weed management practices in transplanted rice by using glyphosate. Biennial Conference on "Recent Advances in Weed Science Research-2010", Indira Gandhi Krishi Vishwavidyalaya, Raipur (Chhatisgarh). pp. 68.

Reddy, C. N., Reddy, M. D and Devi, M. P. Effect of cinosulfuron on weeds and transplanted kharif rice. Indian $J$ of Weed Sci. 2003; 35(1\&2):117-118.

Singh, M. and Singh, R. P. Efficacy of herbicides under different methods of direct seeded rice establishments. Indian J.Agric. Sci., 2010, 80:815-819

Smith, R.J. Control of red rice in water seeded rice (Oryza sativa). Weed Sci., 1981, 29: 663-666Suryakala, P., G Murugan, G., Saravanaperumal, M., Suseendran, K. and P Stalin. Effect of weed management practices with new generation herbicides in transplanted rice. Journal of Pharmacognosy and Phytochemistry, 2019a, 8(3): 39133915.

Suryakala, P., Murugan, G., Stalin, P., Saravanaperumal, M. and Suseendran, $\mathrm{K}$. Effect of pre and post emergence herbicides on weed flora and yield of 
transplanted Rice. Plant Archives,2019b,19(2) : 3093-3096

Vivek Yadav and Bhagwan Singh. Effect of crop Advances establishment method and weed management practices on rice ad associated weeds. Indian $\mathbf{J}$ Argon. 2006; 51(4):301-303.

Upasani, R. R. and Barla, S. Weed control methods in direct seeded rice under medium land condition. Journal of Crop and Weed, 2014, 10(2):445-450.

Yadav D. B., Yadav A and Punia, S. S. Evaluation of bispyribacsodium for weed control in transplanted rice. Indian Journal of Weed Science, 2009,
41(1\&2): 23-27.

Yadav, D. B., Yadav, A., Punia, S. S., Singh, N. and Anil Duhan. Pretilachlor + pyrazosulfuron-ethyl (ready-mix) against complex weed flora in transplanted rice and its residual effects. Indian Journal of Weed Science, 2018, 50 (3): 257-261.

Yadav, D. B., Yadav, A., Punia, S. S. Effectiveness of triafamone + ethoxysulfuron (pre-mix) against complex weed flora in transplanted rice and its residual effects on wheat. Indian Journal of Weed Science, 2019, 51(2), 106-110.

\section{How to cite this article:}

Shivashenkaramurthy, M., Roopa S. Patil, M. J. Manju, K. S. Krishna and Annapurna Neeralagi. 2021. Effect of New Generation Ready-Mix Herbicide against Complex Weed Flora in Transplanted Paddy and its Residual Effect on Succeeding Black Gram Crop. Int.J.Curr.Microbiol.App.Sci. 10(05): 472-488. doi: https://doi.org/10.20546/ijcmas.2021.1005.055 